\title{
On the limit between short and long GRBs
}

\author{
M. Tarnopolski ${ }^{1}$
}

Received: 15 June 2015 / Accepted: 30 July 2015 / Published online: 11 August 2015

(C) The Author(s) 2015. This article is published with open access at Springerlink.com

\begin{abstract}
Two classes of GRBs have been identified thus far without doubt and are prescribed to different physical scenarios-NS-NS or NS-BH mergers, and collapse of massive stars, for short and long GRBs, respectively. The existence of two distinct populations was inferred through a bimodal distribution of the observed durations $T_{90}$, and the commonly applied $2 \mathrm{~s}$ limit between short and long GRBs was obtained by fitting a parabola between the two peaks in binned data from BATSE 1B. Herein, by means of a maximum likelihood (ML) method a mixture of two Gaussians is fitted to the datasets from BATSE, Swift, BeppoSAX, and Fermi in search for a local minimum that might serve as a new, more proper, limit for the two GRB classes. It is found that Swift and BeppoSAX distributions are unimodal, hence no local minimum is present, Fermi is consistent with the conventional limit, whereas BATSE gives the limit significantly longer (equal to $3.38 \pm 0.27 \mathrm{~s}$ ) than $2 \mathrm{~s}$. These new values change the fractions of short and long GRBs in the samples examined, and imply that the observed $T_{90}$ durations are detector dependent, hence no universal limiting value may be applied to all satellites due to their different instrument specifications. Because of this, and due to the strong overlap of the two-Gaussian components, the straightforward association of short GRBs to mergers and long ones to collapsars is ambiguous.
\end{abstract}

Keywords Gamma-ray burst: general · Methods: data analysis · Methods: statistical

\footnotetext{
M. Tarnopolski

mariusz.tarnopolski@uj.edu.pl

1 Astronomical Observatory of the Jagiellonian University, ul. Orla 171, 30-244 Kraków, Poland
}

\section{Introduction}

Gamma-ray bursts (GRBs) were detected by military satellites Vela in late 1960's. GRBs were recognized early to be of extrasolar origin (Klebesadel et al. 1973). Mazets et al. (1981) first observed a bimodal distribution of $T_{90}$ (time during which $90 \%$ of the burst's fluence is accumulated) drawn for 143 events detected in the KONUS experiment. Burst and Transient Source Explorer (BATSE) onboard the Compton Gamma Ray Observatory (CGRO) (Meegan et al. 1992) allowed to confirm the hypothesis that GRBs are of extragalactic origin due to isotropic angular distribution in the sky combined with the fact that they exhibited an intensity distribution that deviated strongly from the $-3 / 2$ power law (Briggs 1995; Fishman and Meegan 1995). However, a more complete sample of BATSE short GRBs were shown to be distributed anisotropically (Mészáros and Štoček 2003; Vavrek et al. 2008) and cosmological consequences were discussed lately (Mészáros et al. 2009). BATSE 1B data release was followed by further investigation of the $T_{90}$ distribution (Kouveliotou et al. 1993) that lead to establishing the common classification of GRBs into short $\left(T_{90}<2 \mathrm{~s}\right)$ and long $\left(T_{90}>2 \mathrm{~s}\right)$. This $2 \mathrm{~s}$ limit was derived by fitting a parabola to the local minimum of the binned distribution of 222 GRBs. It was observed that durations $T_{90}$ seem to exhibit log-normal distributions which were fitted to short and long GRBs (McBreen et al. 1994), resulting in mean durations equal to $0.37 \mathrm{~s}$ and $26.36 \mathrm{~s}$. A mixture of Gaussians fitted to $\log T_{90}$ dataset from BATSE $2 \mathrm{~B}$ yielded locations of the components equal to $0.60 \mathrm{~s}$ and $32.1 \mathrm{~s}$ (Koshut et al. 1996), while a subset of BATSE 3B sample yielded $0.42 \mathrm{~s}$ and $34.4 \mathrm{~s}$ (Kouveliotou et al. 1996). A complete BATSE dataset gave mean locations of the groups at $0.78 \mathrm{~s}$ and 34.7 s (Horváth 2002). The progenitors of long GRBs are associated with supernovae (Woosley and Bloom 2006) 
related with collapse of massive, e.g. Wolf-Rayet, stars. Progenitors of short GRBs are thought to be NS-NS or NS-BH mergers (Nakar 2007), and no connection between short GRBs and supernovae has been proven (Zhang et al. 2009).

The existence of an intermediate-duration GRB class, consisting of GRBs with $T_{90}$ in the range 2-10 s, was put forward (Horváth 1998; Mukherjee et al. 1998) based on the analysis of BATSE 3B data. It was supported (Horváth 2002) with the use of the complete BATSE dataset. Evidence for a third normal component was also found in Swift data (Horváth et al. 2008, 2010; Zhang and Choi 2008; Huja et al. 2009). BeppoSAX dataset was shown to be in agreement with earlier results regarding the bimodal distribution, and the detection of an intermediate-duration component was established on a lower, compared to BATSE and Swift, significance level due to a less populate sample (Horváth 2009). It is important to note that in BeppoSAX only the intermediate and long GRBs were detected, the short ones being not present. Interestingly, Zitouni et al. (2015) re-examined the BATSE current catalog as well as the Swift dataset, and found that a mixture of three Gaussians fits the Swift data better than a two-Gaussian, while in the BATSE case statistical tests did not support the presence of a third component. Regarding Fermi, a three-Gaussian is a better fit than a twoGaussian, ${ }^{1}$ however the presence of a third group in the $T_{90}$ distribution was found to be unlikely (Tarnopolski 2015a,b).

The $2 \mathrm{~s}$ limit is widely used in GRB analysis. However, the Swift data were re-examined (Bromberg et al. 2013) and it was found that a limit of $0.8 \mathrm{~s}$ is more suitable for the GRBs observed by Swift. Many works in which a twoGaussian was fitted to the $\log T_{90}$ distribution showed a significant overlap of components corresponding to short and long GRBs (McBreen et al. 1994; Koshut et al. 1996; Horváth 2002, 2009; Zhang and Choi 2008; Huja et al. 2009; Barnacka and Loeb 2014; Zitouni et al. 2015), regarding datasets from BATSE, Swift, BeppoSAX, Fermi, among others. The mentioned datasets consist of $\sim 1000-2000$ events. Based on the well-established conjecture that durations $T_{90}$ are log-normally distributed, the limit between short and long GRBs may be placed at the position of the local minimum of a mixture distribution.

The aim of this paper is to examine what limits are most suitable for GRB samples observed by different satellites. In Sect. 2, the datasets and methods are described. Results are shown in Sect. 3, while Sect. 4 is devoted to discussion and is followed by concluding remarks gathered in Sect. 5 .

\footnotetext{
${ }^{1}$ Adding parameters to a nested model always results in a better fit (in the sense of a lower $\chi^{2}$ or a higher maximum log-likelihood) due to more freedom given to the model to follow the data. The important question is whether this improvement is statistically significant, and whether the model is an appropriate one. See Tarnopolski (2015a,b) for a discussion.
}

\section{Datasets and methods}

The datasets ${ }^{2}$ from BATSE, ${ }^{3}$ Swift,${ }^{4}$ BeppoSAX,${ }^{5}$ and Fermi ${ }^{6}$ are considered herein. They contain 2041 GRBs (BATSE current catalog), 914 (Swift), 1003 (BeppoSAX), and 1596 (Fermi). Additionally, a subset of BATSE data, i.e. complete BATSE 1B sample containing $226 \mathrm{GRBs}$, is examined to compare with results of Kouveliotou et al. (1993). For display purposes, histograms are plotted using the Knuth rule for bin width. Up to date, to the best of the author's knowledge, only Horváth et al. (2012) and Qin et al. (2013) conducted research on a Fermi subsample, consisting of 425 GRBs from the first release of the catalog.

The fittings are performed using the maximum likelihood (ML) method (Kendall and Stuart 1973). Having a distribution with a probability density function (PDF) given by $f=f(x ; \theta)$ (possibly a mixture), where $\theta=\left\{\theta_{i}\right\}_{i=1}^{p}$ is a set of $p$ parameters, the log-likelihood function is defined as

$\mathcal{L}=\sum_{i=1}^{N} \log f\left(x_{i} ; \theta\right)$,

where $\left\{x_{i}\right\}_{i=1}^{N}$ are the datapoints from the sample to which a distribution is fitted. The fitting is performed by searching a set of parameters $\theta$ for which the $\log$-likelihood $\mathcal{L}$ is maximized. The fitted function in this case is a mixture of two standard Gaussians, $\mathcal{N}\left(\mu, \sigma^{2}\right)$ :

$$
\begin{aligned}
f_{k}(x) & =\sum_{i=1}^{k} A_{i} \varphi\left(\frac{x-\mu_{i}}{\sigma_{i}}\right) \\
& =\sum_{i=1}^{k} \frac{A_{i}}{\sqrt{2 \pi} \sigma_{i}} \exp \left(-\frac{\left(x-\mu_{i}\right)^{2}}{2 \sigma_{i}^{2}}\right) .
\end{aligned}
$$

Here, $k=2$, so the distribution is described by $p=5$ parameters: two means $\mu_{1}, \mu_{2}$, two dispersions $\sigma_{1}, \sigma_{2}$, and one weight $A_{1}$. The second weight is $A_{2}=1-A_{1}$ due to normalization. Normal distribution's PDF is denoted by $\varphi$.

To estimate the parameter errors, $\delta \theta$, a simple Monte Carlo technique called a parametric bootstrap (Efron 1979, 1981; Efron and Tibshirani 1994) is performed, i.e., having a distribution from Eq. (2) fitted, it is randomly sampled to create a set of $N$ random variates ( $N$ being the same as in the original dataset). This set is used to find another fit. After repeating this procedure 1000 times, the standard deviations are computed from the 1000 sets of 5 parameters, and serve as errors for the parameters obtained from the original dataset.

\footnotetext{
${ }^{2}$ All accessed on April 29, 2015.

${ }^{3}$ http://gammaray.msfc.nasa.gov/batse/grb/catalog/current.

${ }^{4}$ http://swift.gsfc.nasa.gov/archive/grb_table.

${ }^{5}$ https://heasarc.gsfc.nasa.gov/docs/sax/sax.html.

${ }^{6} \mathrm{http}: / /$ heasarc.gsfc.nasa.gov/W3Browse/fermi/fermigbrst.html.
} 
In the same manner the error of the location of the local minimum is estimated. In this case, some of the realizations drawn from the original bimodal distribution may happen to be unimodal, hence no local minimum might be present. In that case, only a fraction of the 1000 realizations which do have a local minimum is taken into account. The opposite situation may also occur, i.e. sampling a unimodal distribution and executing the bootstrap may result in some bimodal realizations. However, it turnt out that this kind of situations happen rarely, especially the latter, so no ambiguity is encountered.
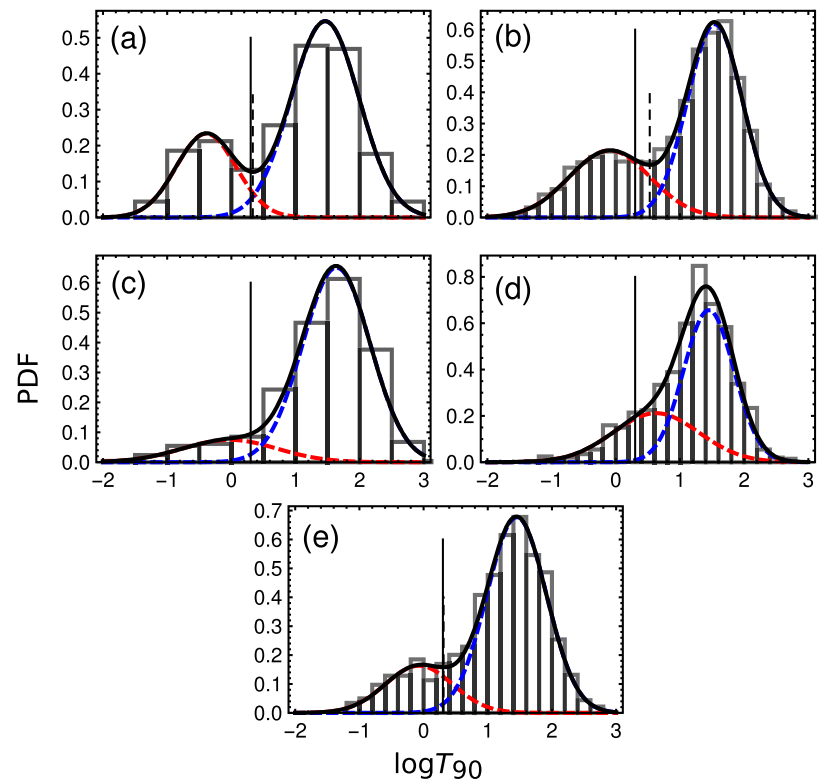

Fig. 1 Two-Gaussian PDFs fitted to $\log T_{90}$ data. Color dashed curves are the components of the (solid black) mixture distribution. Vertical solid line marks the conventional $2 \mathrm{~s}$ limit between short and long GRBs. Vertical dashed line marks the position of the local minimum (if present) of the mixture. The panels correspond to (a) BATSE 1B, (b) BATSE current, (c) Swift, (d) BeppoSAX, and (e) Fermi catalogs. In the latter, the new limit is very close to the conventional limit of Kouveliotou et al. (1993)

\section{Results}

The results in graphical form are displayed in Fig. 1, where the vertical solid line marks the conventional limit of $2 \mathrm{~s}$, and the vertical dashed line marks the location of the minimum of a mixture of two normal distributions (a two-Gaussian). The Swift and BeppoSAX distributions are unimodal, so no new limit may be inferred. Among the 1000 bootstrap executions, 163 realizations were bimodal for the Swift sample, and only one yielded a local minimum in the case of BeppoSAX. Parameters of the fits are gathered in Table 1.

BATSE 1B yielded a minimum at $2.16 \pm 0.05 \mathrm{~s}$, close to the value attained by Kouveliotou et al. (1993). However, for the current BATSE catalog a limit of $3.38 \pm 0.27 \mathrm{~s}$ is more suitable, and the conventional value of $2 \mathrm{~s}$ lies outside the interval more than five times of the error. Fermi dataset is the most consistent with the $2 \mathrm{~s}$ limit, yielding a minimum at $2.05 \pm 0.25 \mathrm{~s}$. The shallower the minimum, the bigger the error obtained.

In the case of BATSE current catalog, all 1000 bootstrap realizations were bimodal; in BATSE $1 \mathrm{~B}$ a minimum was present in 911 cases, while for Fermi there were 896 bimodal realizations.

\section{Discussion}

Bromberg et al. (2013) found, by constructing a PDF for collapsars and non-collapsars (a classification based on physical origin of a GRB), that while the $2 \mathrm{~s}$ criterion is suitable for BATSE, a value of $0.8 \mathrm{~s}$ is more appropriate for the Swift dataset. Herein, the $\log T_{90}$ distributions examined imply that the suitable limit between short and long GRBs for the BATSE current catalog should be $3.38 \mathrm{~s}$, based on a univariate analysis. This is significantly higher than the commonly applied $2 \mathrm{~s}$ criterion. In case of Swift, the duration distribution turnt out to be unimodal, and as such no natural

Table 1 Parameters of the fits. Label corresponds to panels in Fig. 1. Errors are estimated using the bootstrap method

\begin{tabular}{|c|c|c|c|c|c|c|c|c|c|c|c|}
\hline Label & Dataset & $N$ & $i$ & $\mu_{i}$ & $\delta \mu_{i}$ & $\sigma_{i}$ & $\delta \sigma_{i}$ & $A_{i}$ & $\delta A_{i}$ & $\min$. & $\delta \min$. \\
\hline \multirow[t]{2}{*}{ (a) } & \multirow[t]{2}{*}{ BATSE 1B } & \multirow[t]{2}{*}{226} & 1 & -0.393 & 0.099 & 0.465 & 0.069 & 0.272 & \multirow[t]{2}{*}{0.040} & \multirow[t]{2}{*}{2.158} & \multirow[t]{2}{*}{0.049} \\
\hline & & & 2 & 1.460 & 0.056 & 0.532 & 0.044 & 0.728 & & & \\
\hline \multirow[t]{2}{*}{ (b) } & \multirow{2}{*}{$\begin{array}{l}\text { BATSE } \\
\text { current }\end{array}$} & \multirow[t]{2}{*}{2041} & 1 & -0.095 & 0.051 & 0.627 & 0.033 & 0.336 & \multirow[t]{2}{*}{0.018} & \multirow[t]{2}{*}{3.378} & \multirow[t]{2}{*}{0.272} \\
\hline & & & 2 & 1.544 & 0.018 & 0.429 & 0.013 & 0.664 & & & \\
\hline \multirow[t]{2}{*}{ (c) } & \multirow[t]{2}{*}{ Swift } & \multirow[t]{2}{*}{914} & 1 & -0.026 & 0.255 & 0.740 & 0.120 & 0.139 & \multirow[t]{2}{*}{0.042} & \multirow[t]{2}{*}{-} & \multirow[t]{2}{*}{-} \\
\hline & & & 2 & 1.638 & 0.031 & 0.528 & 0.023 & 0.861 & & & \\
\hline \multirow[t]{2}{*}{ (d) } & \multirow[t]{2}{*}{ ВерроSAX } & \multirow[t]{2}{*}{1003} & 1 & 0.626 & 0.186 & 0.669 & 0.075 & 0.355 & \multirow[t]{2}{*}{0.084} & \multirow[t]{2}{*}{-} & \multirow[t]{2}{*}{-} \\
\hline & & & 2 & 1.449 & 0.035 & 0.393 & 0.027 & 0.645 & & & \\
\hline \multirow[t]{2}{*}{ (e) } & \multirow[t]{2}{*}{ Fermi } & \multirow[t]{2}{*}{1596} & 1 & -0.072 & 0.073 & 0.525 & 0.044 & 0.215 & \multirow[t]{2}{*}{0.021} & \multirow[t]{2}{*}{2.049} & \multirow[t]{2}{*}{0.248} \\
\hline & & & 2 & 1.451 & 0.021 & 0.463 & 0.014 & 0.785 & & & \\
\hline
\end{tabular}


Table 2 Fractions of long GRBs and overlap of components of the two-Gaussian fits

\begin{tabular}{llllc}
\hline Label & Dataset & \multicolumn{2}{l}{ Long GRBs fraction [\%] } & Overlap [\%] \\
\cline { 3 - 4 } & & Conventional $^{\mathrm{a}}$ & This work $^{\mathrm{b}}$ & \\
\hline (a) & BATSE 1B & 73.89 & 73.89 & 5.68 \\
(b) & BATSE current & 75.50 & 71.53 & 10.1 \\
(c) & Swift & 90.81 & - & 9.30 \\
(d) & BeppoSAX & 88.24 & - & 34.4 \\
(e) & Fermi & 83.40 & 83.08 & 9.06 \\
\hline
\end{tabular}

${ }^{a}$ When the conventional (Kouveliotou et al. 1993) limit of $2 \mathrm{~s}$ is applied

${ }^{\mathrm{b}}$ When the new limits obtained herein are applied

limit may be inferred. Also BeppoSAX durations are unimodal, giving no new limiting value. It is important to note that the locations $\mu_{1}$, corresponding to the shorter component, are negative (hence $T_{90}<1 \mathrm{~s}$ ) for BATSE (1B and current), Swift and Fermi, while for BeppoSAX it is $\mu_{1}=0.626$, corresponding to $T_{90} \approx 4.23 \mathrm{~s}$. This is definitely not a short GRB group, and it is consistent with Horváth (2009) where the short GRB group was not detected. The Fermi data have a minimum at $2.05 \mathrm{~s}$, consistent with the common limiting value.

The newly obtained limits result in different populations of short and long GRBs in the datasets examined (see Table 2). In BATSE 1B the fraction of long GRBs in the sample is unchanged (due to smallness of the sample; it appears there are no GRBs with durations between $2 \mathrm{~s}$ and $2.16 \mathrm{~s}$ ). In Fermi this fraction is nearly the same, slightly smaller than conventional due to the limit being slightly higher than $2 \mathrm{~s}$. The biggest difference is visible in the BATSE current catalog, where the new limit leads to diminishing the long GRBs fraction by $4 \%$.

All catalogs are dominated by long GRBs, the highest fraction of more than $90 \%$ being observed by Swift. In BATSE current, the proportion of short and long GRBs is $\sim 1: 3$. Swift is more sensitive in soft bands (corresponding to long GRBs) than BATSE was, while Fermi's sensitivity at very soft and very hard GRBs had increased compared to BATSE (Meegan et al. 2009). BeppoSAX is also more sensitive to long GRBs due to the trigger system which used $1 \mathrm{~s}$ as short integration time (Horváth 2009), hence the lack of a distinct short GRB peak. GRBs tend to be softer at later times, hence the inferred duration is shorter than it might be. This naturally leads to a conclusion that the duration distributions as observed by different satellites must differ between each other, and also the limit between short and long GRBs (the local minimum) has to be placed at different locations.

The duration $T_{90}$ itself is not an unambiguous indicator of a GRB type, as the components of the fitted two-Gaussians overlap strongly. To quantify this overlap, the common area under the curves is computed (the total area of a twoGaussian PDF is equal to unity, and the area under each component is given by the weights $A_{i}$ ). This gives a probability of misclassifying a GRB from $5.68 \%$ (BATSE 1B) to $10.1 \%$ (BATSE current), and an enormous $34.4 \%$ in the case of BeppoSAX (see Table 2).

A solution, proposed to deal with the classification ambiguity problem, was proposed and examined in a number of papers (Hakkila et al. 2003; Horváth et al. 2004, 2006, 2010; Chattopadhyay et al. 2007; Veres et al. 2010). The idea is to examine a multi-dimensional space of various parameters; particularly, a two-dimensional space of the hardness ratio vs. duration $T_{90}$. This approach still awaits to be applied to the Fermi GRBs. Additional parameters have been defined and proposed for GRB classification as well. Examples are $\varepsilon=E_{\gamma, \text { iso }, 52} / E_{p, z, 2}^{5 / 3}$ (unambiguously dividing short and long GRBs) (Lü et al. 2010), minimum variability timescale (MVTS) (Bhat 2013; MacLachlan et al. 2012, 2013a,b; Golkhou and Butler 2014; Golkhou et al. 2015) or Hurst exponent (HE) (MacLachlan et al. 2013b; Tarnopolski 2015c). Still, the most common criterion is the GRB duration, and its limitting value has been shown herein to be detector dependent.

\section{Conclusions}

The duration distributions of various catalogs (BATSE 1B, BATSE current, Swift, BeppoSAX, and Fermi) were examined. A mixture of two Gaussians was fitted to the $\log T_{90}$ distributions in search for a new limiting value placed at the local minimum. It was found that the datasets from Swift and BeppoSAX are unimodal, hence no new limit may be inferred. The results from BATSE 1B and Fermi are consistent with the conventional phenomenological limit of $2 \mathrm{~s}$ (Kouveliotou et al. 1993), whereas in BATSE current catalog the value obtained is equal to $3.38 \pm 0.27 \mathrm{~s}$. This leads to a different than commonly established, fraction of long GRBs in the sample, diminished by $4 \%$ (see Table 2).

Due to the significant overlap and dependence of the location of the minimum on the detector, while the division into short and long GRBs based on their durations is qualitatively proper, it is not unambiguously related to its progenitor, i.e. collapsar or non-collapsar. Therefore, as the shortlong phenomenological classification justifies the existence of two distinct GRB classes, it gives limited insight into the underlying physical phenomenon.

Open Access This article is distributed under the terms of the Creative Commons Attribution 4.0 International License (http://creative commons.org/licenses/by/4.0/), which permits unrestricted use, distribution, and reproduction in any medium, provided you give appropriate credit to the original author(s) and the source, provide a link to the Creative Commons license, and indicate if changes were made. 


\section{References}

Barnacka, A., Loeb, A.: Astrophys. J. 794, L8 (2014)

Bhat, P.N.: In: Castro-Tirado, A.J., Gorosabel, J., Park, I.H. (eds.) Temporal Decomposition Studies of GRB Lightcurves. EAS Publ. Ser., vol. 61, p. 45. Cambridge University Press, Cambridge (2013)

Briggs, M.S.: Astrophys. Space Sci. 231, 3 (1995)

Bromberg, O., Nakar, E., Piran, T., Sari, R.: Astrophys. J. 764, 179 (2013)

Chattopadhyay, T., Misra, R., Chattopadhyay, A.K., Naskar, M.: Astrophys. J. 667, 1017 (2007)

Efron, B.: Ann. Stat. 7, 1 (1979)

Efron, B.: Biometrika 68, 589 (1981)

Efron, B., Tibshirani, R.J.: An Introduction to the Bootstrap. CRC Press, Boca Raton (1994)

Fishman, G.J., Meegan, C.A.: Annu. Rev. Astron. Astrophys. 33, 415 (1995)

Golkhou, V.Z., Butler, N.R.: Astrophys. J. 787, 90 (2014)

Golkhou, V.Z., Butler, N.R., Littlejohns, O.M.: Astrophys. J. (2015, in press). arXiv: 1501.05948

Hakkila, J., Giblin, T.W., Roiger, R.J., Haglin, D.J., Paciesas, W.S., Meegan, C.A.: Astrophys. J. 582, 320 (2003)

Horváth, I.: Astrophys. J. 508, 757 (1998)

Horváth, I.: Astron. Astrophys. 392, 791 (2002)

Horváth, I.: Astrophys. Space Sci. 323, 83 (2009)

Horváth, I., Mészáros, A., Balázs, L.G., Bagoly, Z.: Balt. Astron. 13, 217 (2004)

Horváth, I., Balázs, L.G., Bagoly, Z., Ryde, F., Mészáros, A.: Astron. Astrophys. 447, 23 (2006)

Horváth, I., Balázs, L.G., Bagoly, Z., Veres, P.: Astron. Astrophys. 489, L1 (2008)

Horváth, I., Bagoly, Z., Balázs, L.G., de Ugarte Postigo, A., Veres, P., Mészáros, A.: Astrophys. J. 713, 552 (2010)

Horváth, I., Balázs, L.G., Hakkila, J., Bagoly, Z., Preece, R.D.: PoS (GRB2012), 046 (2012)

Huja, D., Mészáros, A., Řípa, J.: Astron. Astrophys. 504, 67 (2009)

Kendall, M., Stuart, A.: The Advanced Theory of Statistics. Griffin, London (1973)

Klebesadel, R.W., Strong, I.B., Olson, R.A.: Astrophys. J. 182, L85 (1973)

Koshut, T.M., Paciesas, W.S., Kouveliotou, C., van Paradijs, J., Pendleton, G.N., Fishman, G.J., Meegan, C.A.: Astrophys. J. 463, 570 (1996)

Kouveliotou, C., Meegan, C.A., Fishman, G.J., Bhat, N.P., Briggs, M.S., Koshut, T.M., Paciesas, W.S., Pendleton, G.N.: Astrophys. J. 413, L101 (1993)

Kouveliotou, C., Koshut, T., Briggs, M.S., Pendleton, G.N., Meegan, C.A., Fishman, G.J., Lestrade, J.P.: AIP Conf. Proc. 384, 42 (1996)
Lü, H.-J., Liang, E.-W., Zhang, B.-B., Zhang, B.: Astrophys. J. 725, $1965(2010)$

MacLachlan, G.A., Shenoy, A., Sonbas, E., Dhuga, K.S., Eskandarian, A., Maximon, L.C., Parke, W.C.: Mon. Not. R. Astron. Soc. 425, L32 (2012)

MacLachlan, G.A., et al.: Mon. Not. R. Astron. Soc. 432, 857 (2013a)

MacLachlan, G.A., Shenoy, A., Sonbas, E., Coyne, R., Dhuga, K.S., Eskandarian, A., Maximon, L.C., Parke, W.C.: Mon. Not. R. Astron. Soc. 436, 2907 (2013b)

Mazets, E.P., Golenetskii, S.V., Ilinskii, V.N., Panov, V.N., Aptekar, R.L., Gurian, I.A., Proskura, M.P., Sokolov, I.A., Sokolova, Z.I., Kharitonova, T.V.: Astrophys. Space Sci. 80, 3 (1981)

McBreen, B., Hurley, K.J., Long, R., Metcalfe, L.: Mon. Not. R. Astron. Soc. 271, 662 (1994)

Meegan, C.A., Fishman, G.J., Wilson, R.B., Horack, J.M., Brock, M.N., Paciesas, W.S., Pendleton, G.N., Kouveliotou, C.: Nature 355, 143 (1992)

Meegan, C.A., Lichti, G., Bhat, P.N., Bissaldi, E., Briggs, M.S., Connaughton, V., Diehl, R., Fishman, G., Greiner, J., Hoover, A.S., van der Horst, A.J., von Kienlin, A., Kippen, R.M., Kouveliotou, C., McBreen, S., Paciesas, W.S., Preece, R., Steinle, H., Wallace, M.S., Wilson, R.B., Wilson-Hodge, C.: Astrophys. J. 702, 791 (2009)

Mészáros, P., Štoček, J.: Astron. Astrophys. 18, 293 (2003)

Mészáros, A., Balázs, L.G., Bagoly, Z., Veres, P.: Balt. Astron. 18, 293 (2009)

Mukherjee, S., Feigelson, E.D., Jogesh Babu, G., Murtagh, F., Fraley, C., Raftery, A.: Astrophys. J. 508, 314 (1998)

Nakar, E.: Phys. Rep. 442, 166 (2007)

Qin, Y., Liang, E.-W., Liang, J.-F., Yi, S.-X., Lin, L., Zhang, B.-B., Zhang, J., Lü, H.-J., Lu, R.-J., Lü, L.-Z., Zhang, B.: Astrophys. J. 763, 15 (2013)

Tarnopolski, M.: Astron. Astrophys. (2015a, in press). arXiv:1506. 07324

Tarnopolski, M.: Preprint (2015b). arXiv:1506.07801

Tarnopolski, M.: Preprint (2015c). arXiv:1507.04886

Vavrek, R., Balázs, L.G., Mészáros, A., Horváth, I., Bagoly, Z.: Mon. Not. R. Astron. Soc. 391, 1741 (2008)

Veres, P., Bagoly, Z., Horváth, I., Mészáros, A., Balázs, L.G.: Astrophys. J. 725, 1955 (2010)

Woosley, S.E., Bloom, J.S.: Annu. Rev. Astron. Astrophys. 44, 507 (2006)

Zhang, Z.-B., Choi, C.-S.: Astron. Astrophys. 484, 293 (2008)

Zhang, B., Zhang, B.-B., Virgili, F.J., Liang, E.-W., Kann, D.A., Wu, X.-F., Proga, D., Lv, H.-J., Toma, K., Mészáros, P., Burrows, D.N., Roming, P.W.A., Gehrels, N.: Astrophys. J. 703, 1696 (2009)

Zitouni, H., Guessoum, N., Azzam, W.J., Mochkovitch, R.: Astrophys. Space Sci. 357, article id. 7 (2015) 